\title{
Submandibular Sialolithiasis: A Brief Overview and Report of Two Cases
}

\author{
Ujjwal Gulati ${ }^{*}$, Rajesh Kshirsagar ${ }^{2}$, Gurkeerat Singh ${ }^{3}$ and Mehvash Gulati ${ }^{4}$ \\ ${ }^{1}$ Consultant Maxillofacial Surgeon, Indiadens, India \\ ${ }^{2}$ Professor \& HOD, Department of Oral and Maxillofacial Surgery, Bharati Vidyapeeth Dental College and Hospital, India \\ ${ }^{3}$ Department of Orthodontics, Sudha Rastogi College of Dental Sciences, India \\ ${ }^{4}$ Chief Consultant and General Dentist, Indiadens, India \\ *Corresponding author: Ujjwal Gulati, Consultant Maxillofacial Surgeon, Indiadens, New Delhi, India

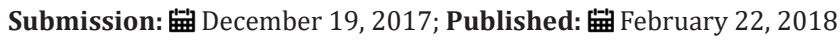

\begin{abstract}
Sialoliths are second most common anomalies of the salivary glands. They are most commonly seen in submandibular glands due to various anatomic and physiologic reasons. They may or may not exhibit clinical signs and symptoms. Also, the visibility on plain radiographs is dependent upon size and degree of calcifications. CT and MRI form a main frame of investigations in current scenario. Surgical removal of calculus intraorally has been the traditional approach for management and is still the mainstay of most surgeons. However, recent advances into the field of lithotripsy and endoscopy have revolutionized the management of salivary calculi. Recovery of the involved gland after removal of the calculi is not predictable.

Keywords: Obstructive sialadenitis; Recurrent sialadenitis; Sialolith; Submandibular gland; Submandibular gland sialadenitis
\end{abstract}

\section{Introduction}

Sialolithiasis is the formation of calcific concretions within the ductal system of a major or minor salivary gland. It is the second most common disease of the salivary glands after mumps. The peak incidence is between the ages of 30 to 50 years. It is seen twice more frequently in men as compared to women. Amongst salivary glands, submandibular gland is the most commonly involved gland with an incidence of $80 \%$ of salivary stones. The submandibular sialolith may be classified based upon its anatomical location; Intra-glandular and extra-glandular. The extra-glandular sialoliths are further classified as anterior sialoliths (situated anterior to the line joining mesial surfaces of mandibular second molars; and posterior sialoliths (those situated in the duct posterior to this line till the extra-glandular part of the duct). The location of a calculus in case of submandibular gland is mostly extra-glandular [1-3].

\section{Etiopathogenesis}

a) Neurohumoral condition causing salivary stagnation $[1,3]$.

b) A central nidus for stone formation $[1,3]$.

c) Metabolic mechanism favouring precipitation of salivary salts into the matrix in the presence of coexisting inflammation $[1,3]$.

\section{Reasons for common involvement of submandibular gland}

pH of saliva: The salivary $\mathrm{pH}$ in submandibular gland is alkaline (6.8-7.1) favouring precipitation of calcium salts [1,4].

Calcium content: Submandibular gland contains relatively higher concentration of calcium and phosphate salts in form of apatites $[1,4]$.

Viscosity: The submandibular gland expresses more viscous saliva due to higher mucous content $[1,4]$.

Anatomic factors: The submandibular duct drains saliva against the gravity as the gland is situated lower than the ductal orifice, contributing to stagnation. Additionally, the ductal course is long and tortuous. The lingual nerve is also held responsible for possible kinking of the duct as it crosses the duct. There is also a possible kinking/bend at the region where the duct passes over the posterior border of mylohyoid muscle $[1,4]$.

\section{Structure and Composition of a Sialolith}

Sialoliths can vary in size and shape. They may be noncalcified mucous plugs (commonly seen in parotid gland) or large concretions which are round/oval/irregularly shaped (seen in 
submandibular gland) [5]. This concentric macroscopic structure of a sialolith supports the theory of a central nidus (bacterial colonization/foreign body/sloughed cells/inflammation) around which calcification occurs in lamellar pattern. The colour may vary from chalky white to pale yellow or even orange, depending upon their organic matrix. The surface may be smooth or rough. If manipulated with force, they easily disintegrate like a chalk. They are mainly composed of calcium phosphate with traces of magnesium and ammonia with an organic matrix consisting of carbohydrates and amino acids $[1,3,6]$.

\section{Signs and Symptoms}

Sialoliths may or may not be symptomatic and the size of the stone may be disproportionate to the severity of symptoms [6]. In other words, a sialolith may reach significant size before eliciting any symptoms. This is more common in case of posterior sialoliths $[2,7] .65 \%$ of patients with recurrent sialadenitis have had sialoliths in the past. Pre and post meal salivary stimulation is frequently associated with stagnation of the saliva and hence swelling in submandibular region. In fact, this is a pathognomic sign of salivary stone $[1,2,8]$. This stagnation may elicit mild discomfort or even moderate levels of pain. The calculus is rarely capable of blocking the duct completely due to relatively larger lumen and elasticity of Wharton's duct, so as the salivation decreases after meals, the stagnated saliva is gradually expressed out causing the swelling to subside. If the calculus is not removed, chronic and recurrent process may lead to chronic recurrent sialadenitis or acute suppurative sialadenitis. This may causes severe exacerbation of symptoms along with systemic manifestations in form of fever, malaise and mild to moderate leukocytosis. Swelling, tenderness and redness may be seen along the course of the duct. The gland is also enlarged, tense and tender. Palpation, especially bimanual, causes severe pain with expression of pus at ductal orifice. However, this acute phase may also lead to spontaneous exfoliation of the calculi as the inflammatory process regards the calculus as a foreign body and tries to get rid of it [1,2].

\section{Clinical Examination}

Submandibular region is inspected for any swelling and palpated for eliciting possible tenderness. The ductal orifice is observed for redness or edema. Palpation along the course of the duct may reveal and locate presence of a salivary stone. The glandular secretions from the ductal orifice are observed. Mild turbid discharge followed by clear saliva is more indicative of inflamed gland and duct due to backpressure and stasis. However, purulent discharge along with moderate to severe tenderness is indicative of infection. There may be no salivary expression in case of complete obstruction of the lumen, though it is very rare $[1,2]$.

\section{Radiographic examination}

The diagnosis of sialolith is based on patient's history and clinical examination. However, radiographic modalities are helpful in localizing the sialolith and determine its size and number.
Plain films: Detection of a sialolith on a radiograph is not only dependent upon its size and degree of calcification, but also on the projection used for capturing the calculus. $80-90 \%$ of submandibular sialoliths are radiopaque enough to appear on radiographs. Three main views are used to detect submandibular calculi. 1) Mandibular occlusal view: It can detect calculi in anterior two-thirds of the duct; 2) 15 degree lateral oblique view: It can detect calculi in posterior one third and intra-glandular portion of the duct; and 3) Orthopantomogram: This is used in case of lack of expertise or availability in obtaining the required projection. The sialolith may also be an incidental finding on an OPG as it is the most frequently used extra-oral projection in dentistry [1,9-11].

Sialography: It is used when calculi are not witnessed on radiographs but clinically obstruction is evident. It may reveal radiolucent calculi in form of a "filling defect" or ductal narrowing on a sialogram $[9,12,13]$.

Computed tomography: It is an excellent modality to localize and estimate the size of the calculi. It is relatively much more sensitive than plain radiographs to detect the presence of even less mineralized stones $[1,9,13]$.

Magnetic resonance imaging: It is more useful to study the glandular architechture and ductal morphology. It is extremely sensitive for any soft tissue mass/tumour causing obstruction and hence chronic sialadenitis (ruling out other causes of sialadenitis). It is relatively less sensitive in locating the stone but may help in detecting strictures, ductal obstructions other than sialoliths and in decision making for submandibular gland removal by estimating the glandular damage $[1,9,13]$.

Ultrasonography: USG has been recently applied widely in detection of many anomalies. It has the propensity for gross detection of size and glandular structure. It may or may not pick sialoliths [14].

\section{Differential diagnosis}

Clinically, the obstruction in salivary flow may also be due to ulcerative papillary obstruction, ductal strictures or a mass adjacent to or invading the duct. Radiographically (plain films), the calculus must be differentiated from calcified lymph nodes, foreign body, phlebolith, calcification in facial artery and myositis ossificans $[3,15]$.

\section{Treatment}

\section{Conservative approach}

It is feasible in cases of non-adherent stones of small size in anterior third of the duct. The gland can be milked and an effort put in to retrieve the calculus from orifice.

\section{Surgical approach}

The sialolith in Wharton's duct can be approached intraorally. The anterior sialoliths can easily be retrieved under local anaesthesia. For posterior sialoliths, patient needs to be put under general anaesthesia. 


\section{Lithotripsy}

It has been used off late to disintegrate the renal stones into minute pieces which can be expressed out easily. The same technology has been used in salivary calculi but has not gained much popularity $[9,16,17]$.

\section{Endoscopic retrieval}

It is one of the recent advances in the field of sialolith retrieval. However, it is popular and more used in tertiary institutions with regular inflow of salivary gland anomalies or units dedicated to treating exclusively salivary gland patients (for eg. salivary gland centers in developed countries) [9].

\section{Surgical Technique}

\section{Anterior sialoliths}

Adequate local anaesthesia is administered. A suture is passed distal to the expected site of calculus sparing the sublingual veins medially and sublingual gland laterally. Another suture is passed between the orifice and lingual frenum. Traction is applied over both sutures to make the mucosa taut. Incision followed by a combination of blunt and sharp dissection is used to gain access to the submandibular duct which usually appears light grey and has network of capillaries overlying it. Sialolith can be seen through the duct. If the stone can be gently milked out of the duct through orifice, it is relatively more conservative than incising the duct. However, if the stone cannot be milked out, a sling suture is passed underneath the duct to stabilize it. Sialolith is localized and a longitudinal incision given over it to retrieve the stone. If the stone is adherent to the duct wall, it may be gently teased out using a curette. The floor of mouth is sutured back leaving the incised portion of the duct unsutured $[1,18]$.

\section{Posterior sialoliths}

General anaesthesia is advisable for posterior sialoliths to facilitate adequate retraction and surgical access. Incision followed by a combination of sharp and blunt dissection is used to isolate the duct. Sutures are used to mobilize the duct anteriorly. Lingual nerve is identified and protected where it crosses the submandibular duct. Once the lingual nerve is protected, dissection is carried out deeper to access the posterior third of the duct. An assistant is instructed to push up the gland, which makes the upper pole of the gland along with extra-glandular portion of the duct more visible and accessible. In most of the cases, calculus can be located at this stage. A longitudinal incision is placed over the duct and the stone is retrieved. The gland is milked to express saliva and to retrieve any additional stones. However, if calculus cannot be located, longitudinal incision is given to explore the duct along with milking of the gland to help advance the distally located stone. The floor of mouth is sutured with the duct left unsutured $[1,18]$.

\section{Prognosis}

A sialolith causes damage to the gland by applying back pressure and hence inflammation, fibrosis atrophy or infection. If the sialolith has been present for a short time, the gland usually recovers completely. In cases of chronic sialadenitis the gland may partially recover with a part of the gland undergoing fibrosis/ atrophy. However, the patient may not have any clinical symptoms except hardness in submandibular region. Clinical examination reveals decreased salivary flow and USG/MRI reveals fibrosis of a part of a gland with loss of glandular architecture. In worst scenario, the gland may not recover from the damage caused due to back pressure and would need to be surgically removed, commonly seen in cases of long standing sialoliths or acute exacerbation of chronic sialadenitis (suppuration/infection) $[3,19,20]$.

\section{Case Report 1}

A 58 year old female reported with a chief complaint of intermittent swelling in left submandibular region. The swelling was related to meals and persisted for 2-3 hours after food. It was variable in size and occurrence. On examination, there was mild swelling in submandibular region (Figure 1). Ductal orifice was slightly reddish and tender signifying inflammation. Expressed saliva was normal in appearance and consistency but the salivary flow was relatively reduced compared to contra lateral side. Clinical diagnosis of left submandibular sialolith was made. A computerised tomogram revealed a $2.4 \mathrm{~mm}$ radiopaque mass in anterior part of the left Wharton's duct (Figure 2a-2c). However, the calculus was not visible or palpable. The sialolith was removed intra orally under local anesthesia. The surgical technique has already been discussed (Figure 3-7).

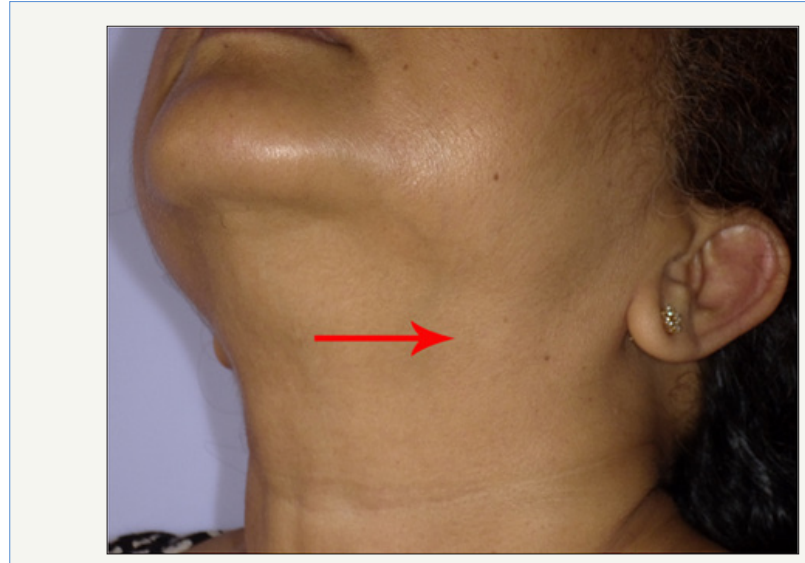

Figure 1: Mild extra-oral swelling.

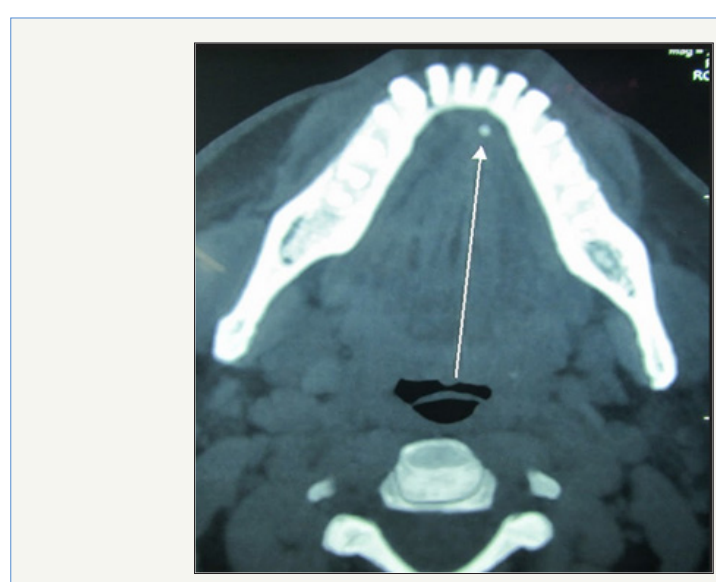

Figure 2a : Axial section of CT showing sialolith 


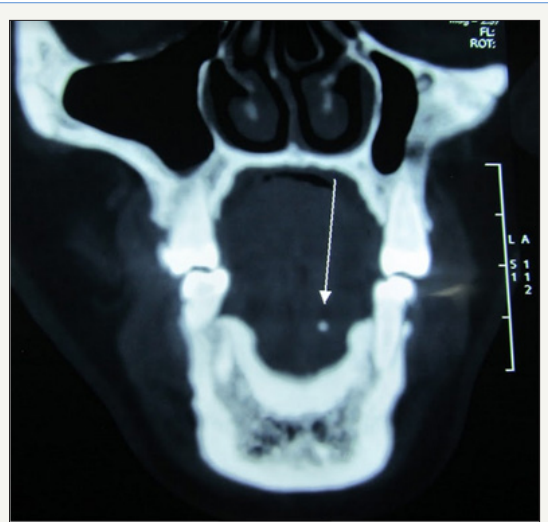

Figure 2b : Coronal section of CT showing sialolith.

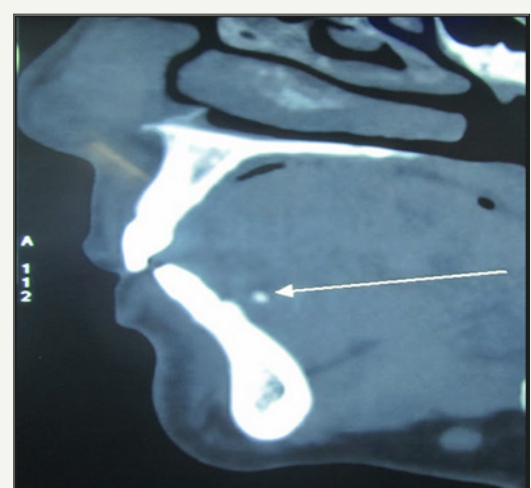

Figure 2c : Sagittal section of CT showing sialolith.

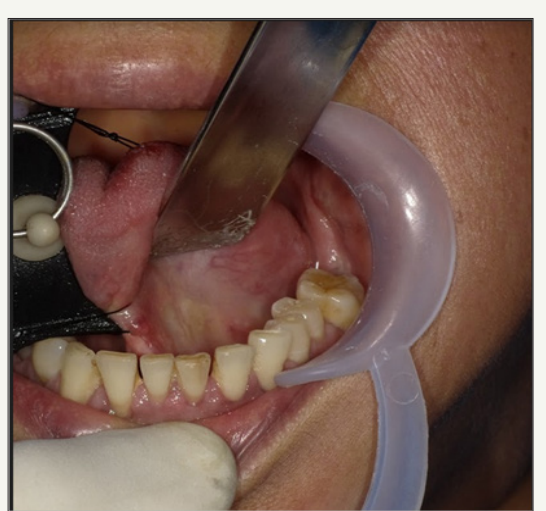

Figure 3 : Tongue and ductal region retracted with sutures.

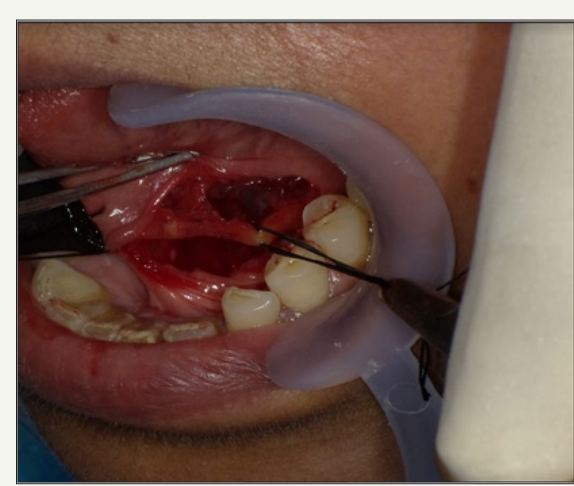

Figure 4: Duct dissected and blocked with a distal suture. Sialolith can be seen within duct.

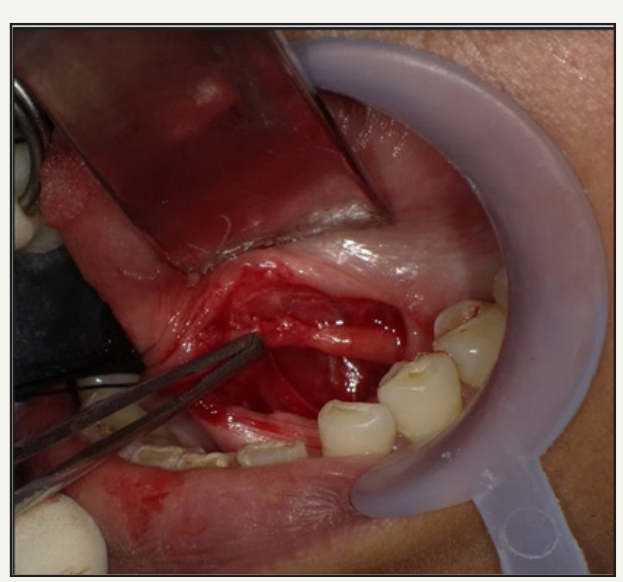

Figure 5 : Duct incised and sialolith retrieved (edge of tissue forcep).

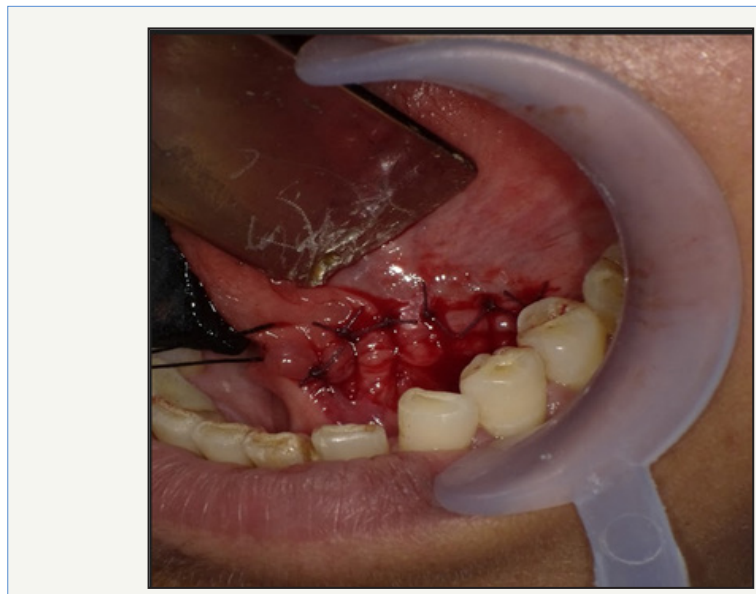

Figure 6 : Suturing with 4-0 vicryl.

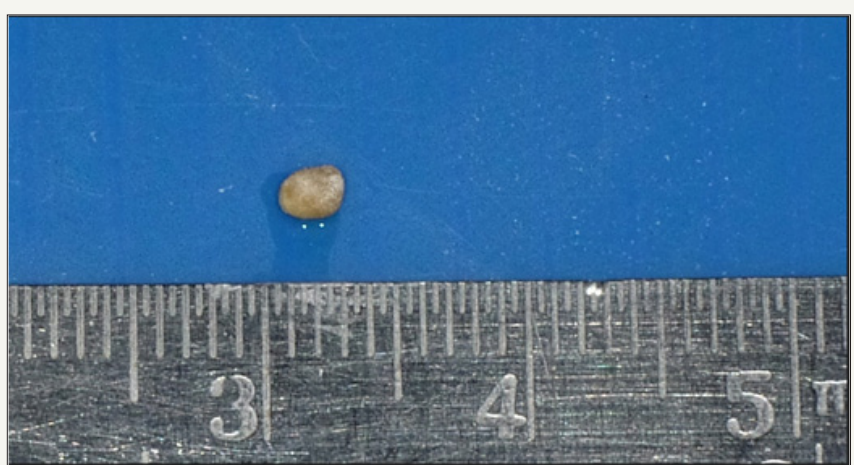

Figure 7 : Retrieved sialolith.

\section{Case Report 2}

A young female reported with a complaint of painful swelling in the right submandibular region (Figure 8). The swelling was present since four to five months. Initially, the swelling was intermittent and was associated with meals. Swelling persisted for one to two hours after food and then gradually subsided. The patient consulted a maxillofacial surgeon and was diagnosed with right submandibular sialolith in posterior portion of the duct and was advised for surgical removal of the sialolith. Since 
the patient was residing away from home (job placement), so she opted for removal at her hometown. When the patient reported to us (delay of about 1 month since consultation with surgeon), she complained of persistent and painful swelling. On examination, right submandibular region was swollen and tender on palpation. Slightly turbid fluid was expressed from ipsilateral Wharton's duct orifice on glandular massage. Salivary flow was absent. Orifice was normal in appearance. A computerised tomogram was already done and showed a sialolith of $6.1 \mathrm{~mm}$ in right posterior Wharton's duct (Figure 9a-9c). Patient was prescribed broad spectrum antibiotics and the surgical removal was planned under general anaesthesia.

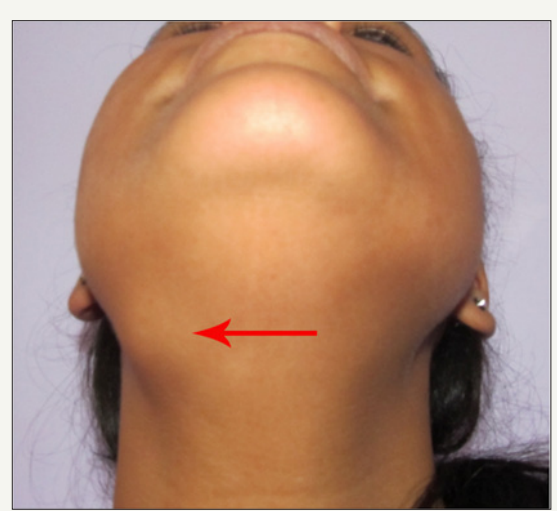

Figure 8 : Preoperative worm's view showing swelling in right submandibular region.

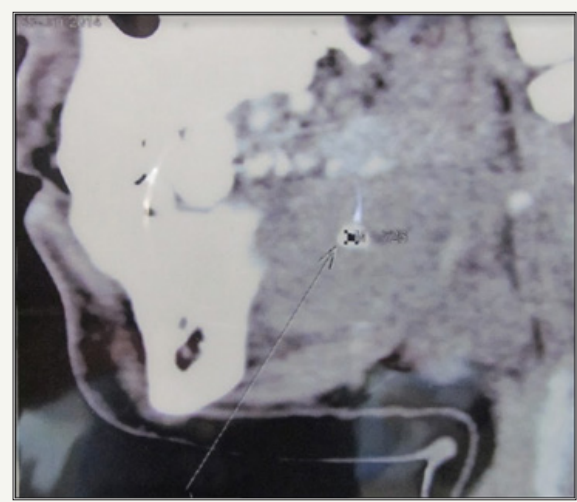

Figure 9a : Sagittal section showing presence of a radiopaque mass.

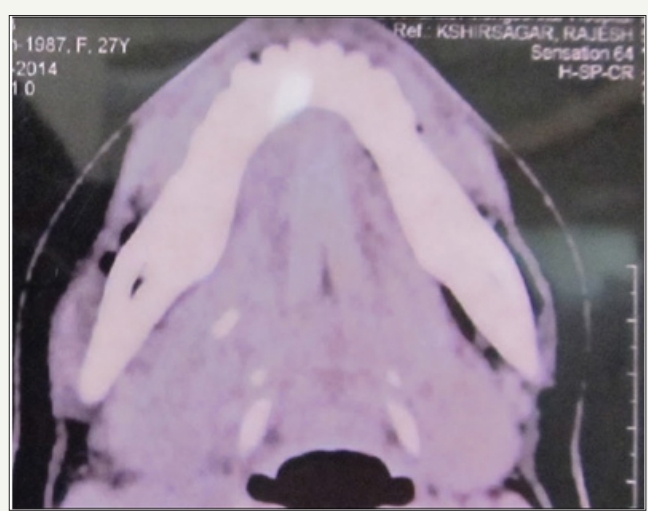

Figure 9b : Axial section showing the location of the radiopaque mass to be behind the third molar region.

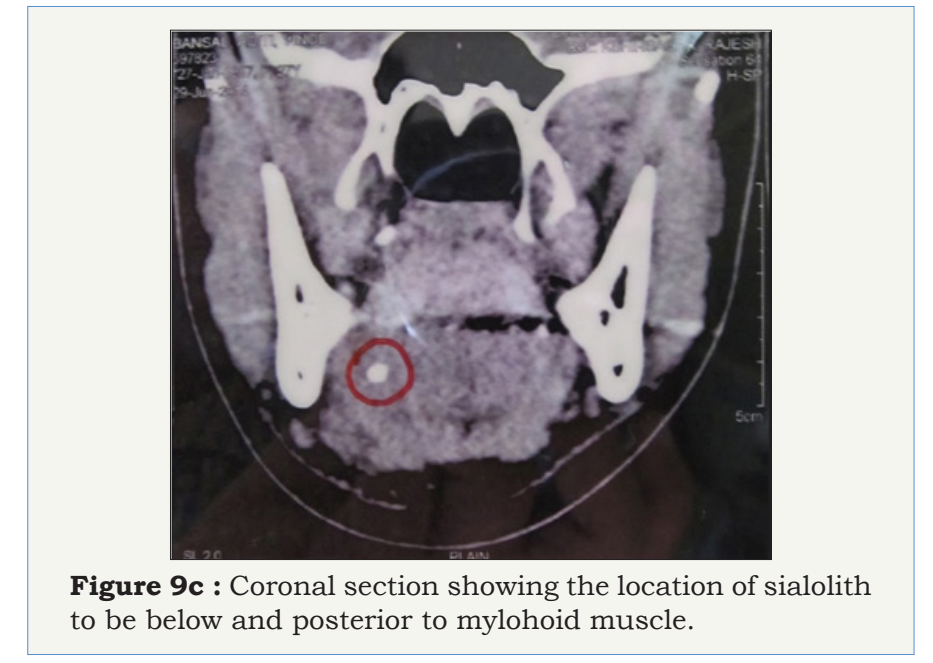

\section{Surgical technique}

After appropriate preparation and draping, a mouth prop was inserted on ipsilateral side and tongue retracted with a suture. An incision was given with an imaginary line running across the duct with a lateral inclination anteriorly and medial inclination posteriorly. This is done to oppose the path of lingual nerve which is exactly opposite to the incision line, i.e. lingual nerve crosses the duct from below, lateral to medial, in premolar-molar region. A combination of blunt and sharp dissection was used to expose the anterior duct. The point of lingual nerve traversing the duct was identified and lingual nerve protected. Duct was further dissected posteriorly. However, the ductal stone could not be visualised. On applying extraoral pressure to push the deeper part of duct intraorally, the sialolith was seen. Duct posterior to the obstruction was edematous. As an incision was given in the duct over the stone, a spurt of pus threw the stone out (Figure $10 \& 11$ ). Gland was further milked and resulted in expression of more pus. Milking was continued till normal salivary flow was observed. The floor of mouth was sutured with 4-0 resorbable suture. Patient was kept on broad spectrum antibiotics and discharged after 2 days. Recovery was uneventful. However, salivary flow was close to nil for about 3 weeks. Patient has been followed up for around 8 months. Some part of the gland has become fibrosed and salivary flow has increased but still less as compared to opposite side.

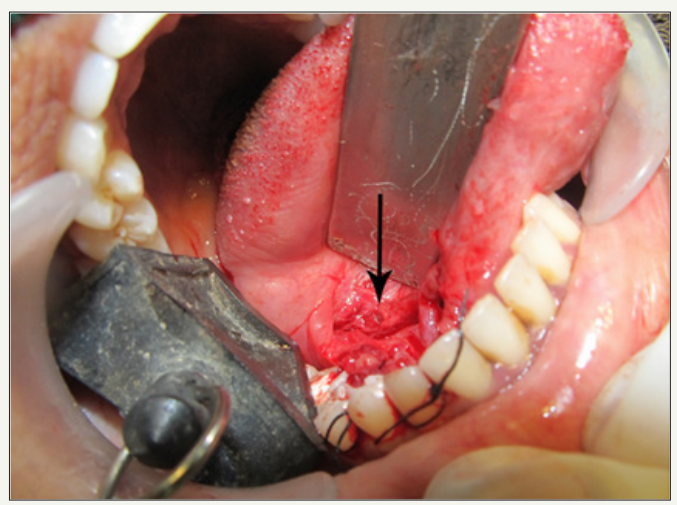

Figure 10 : Operative picture showing the incised part of the duct. 


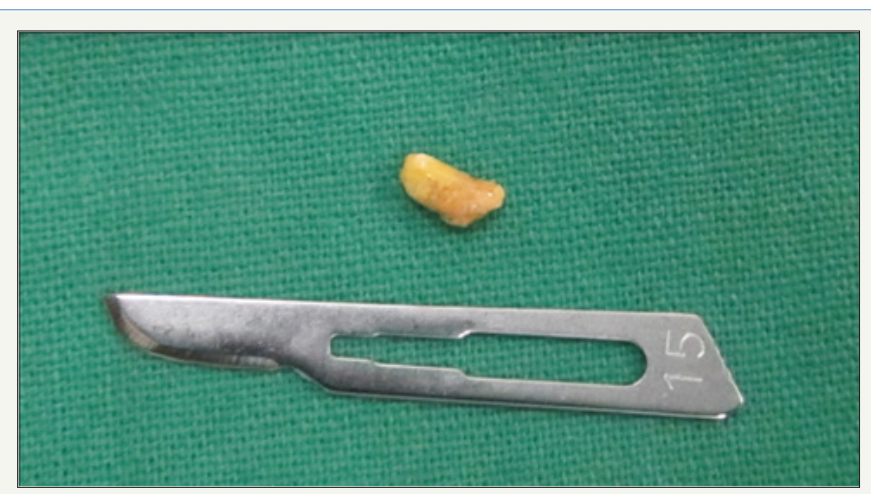

Figure 11 : The retrieved sialolith.

\section{Discussion}

The first case is of a short term sialolith in anterior portion of the duct causing partial obstruction. The salivary flow in this case was reduced as compared to the first case, where it was nil. Differences in duration of illness, persistence of swelling and tenderness of gland should also be appreciated. Complete recovery of the gland post removal was anticipated and post-operative follow up till now has been consistent with expectations.

The second case can be best described as acute exacerbation of chronic obstructive sialadenitis secondary to sialolith in posterior Wharton's duct. The signs and symptoms were consistent with literature. Post-operative follow-up of the patient has revealed partial recovery of the gland with reduced salivary flow. No followup investigations have been performed. The patient was taken into confidence pre-operative for possibility of partial or no recovery of the gland requiring additional surgery for submandibular gland removal. However, the patient has no clinical complaint except slight firmness in submandibular region on palpation.

\section{Conclusion}

History and clinical examination play a vital role in diagnosis of obstructive sialadenitis. Investigations are targeted to rule out rare causes other than sialoliths for obstructive sialadenitis. Also, they help in localizing and determining the size and number of the sialoliths. Anticipated prognosis depends upon size number and location of sialolith, duration of obstruction and presence or absence of infection. Patients must be informed regarding anticipated recovery and possible course of treatment in adverse outcomes.

\section{References}

1. Feinberg SE (1992) Diagnosis and surgical management of salivary gland disorders. In Peterson LJ (Ed.), Principles of Oral and Maxillofacial Surgery, Volume 2. JB Lippincott, Philadelphia, USA, p. 841.
2. Lustman J, Regev E, Melamed Y (1990) Sialolithiasis: A survey on 245 patients and a review of the literature. Int J Oral Maxillofac Surg 19(3): 135-138.

3. Marx RE, Stern D (2003) Non neoplastic salivary gland diseases. In Oral and Maxillofacial Pathology: A rationale for diagnosis and management. Quintessence Publishing co, Illinois, USA, p. 519.

4. Huoh KC, Eisele DW (2011) Etiologic factors in Sialolithiasis. Otolaryngol Head Neck Surg 145(6): 935-939.

5. Suleiman SI, Hobsley M (1980) Radiological appearance of parotid calculi. Br J Surg 67(12): 879-880.

6. Rust TA, Messerly CD (1969) Oddities of salivary calculi. Oral Surg Oral Med Oral Pathol 28(6): 862-865.

7. Brusati R, Fiamminghi L (1973) Large calculus of the submandibular gland: Report of a case. J Oral Surg 31(9): 710-711.

8. Levy BM, ReMine WH, Devine KD (1962) Salivary gland calculi: Pain, swelling associated with eating. JAMA 181: 1115-1119.

9. Katz P, Fritsch MH (2003) Salivary stones: Innovative techniques in diagnosis and treatment. Curr Opin Otolaryngol Head Neck Surg 11(3): 173-178.

10. Massouh H, Dunscombe P (1982) Panoramic Sialography: An alternative technique. Br J Radiol 55(658): 735-739.

11. Morris CR, Marano PD, Swimley DC, Runco JG (1969) Abnormalities noted on panoramic radiographs. Oral Surg Oral Med Oral Pathol 28(5): 772-782.

12. Dalley RW (1994) Sialography-present status: Does it still have a role to play in light of computed tomography, magnetic resonance imaging and ultrasound? In Worthington P, Evans JR (Eds.), Controversies in Oral and Maxillofacial surgery. WB Saunders, Philadelphia, USA, p. 41.

13. Van den Akker HP (1988) Diagnostic imaging in salivary gland disease. Oral Surg Oral Med Oral Pathol 66(5): 625-637.

14. Zenk J, Koch M, Klintworth N, König B, Konz K, et al. (2012) Sialendoscopy in the diagnosis and treatment of Sialolithiasis: A study on more than 1000 patients. Otolaryngol Head Neck Surg 147(5): 858-863.

15. Warpeha RL (1980) Masses in the neck. In: Wood NK, Goaz PW (Eds.), Differential diagnosis of oral lesions. (2nd edn), St. Louis, CV Mosby, USA.

16. Brouns JJ, Hendriks AJ, Birkens AF (1989) Removal of salivary stones with aid of lithotripter. J Craniomaxillofac Surg 17(7): 329-330.

17. Iro H, Waitz G, Nitsche N, Schneider T, Benninger J, et al. (1992) Extracorporeal piezoelectric shock wave lithotripsy of salivary gland stones. Laryngoscope 102(5): 492-494.

18. Park JS, Sohn JH, Kim JK (2006) Factors influencing intraoral removal of submandibular calculi. Otolaryngol Head Neck Surg 135(5): 704-709.

19. Travis LW, Hecht DW (1977) Acute and chronic inflammatory diseases of the salivary glands: Diagnosis and management. Otolaryngol Clin North Am 10(2): 329-338.

20. Yoshimura Y, Inoue Y, Odagawa T (1989) Sonographic examination of Sialolithiasis. J Oral maxillofac surg 47(9): 907-912. 
Creative Commons Attribution 4.0

International License

For possible submissions Click Here

Your subsequent submission with Crimson Publishers will attain the below benefits

- High-level peer review and editorial services

- Freely accessible online immediately upon publication

- Authors retain the copyright to their work

- Licensing it under a Creative Commons license

- Visibility through different online platforms

- Global attainment for your research

- Article availability in different formats (Pdf, E-pub, Full Text)

- Endless customer service

- Reasonable Membership services

- Reprints availability upon request

- One step article tracking system 